\title{
Effects of continuous venovenous hemofiltration on vancomycin trough concentrations in critically ill children
}

\author{
Lengyue Peng ${ }^{1}$, Yawen Gao ${ }^{1}$, Guangli Zhang ${ }^{1}$, Xiaoyin Tian ${ }^{1}$, Huiting $\mathrm{Xu}^{1}$, Qinghong Yu ${ }^{1}$, Jie Cheng ${ }^{1}$, \\ Yuanyuan $\mathrm{Li}^{1}$, Qinyuan $\mathrm{Li}^{1}$, Yingfu Chen ${ }^{2}$, Wei Zhao ${ }^{3}$, Zhengxiu Luo ${ }^{1}$ \\ ${ }^{1}$ Department of Respiratory Medicine Children's Hospital of Chongqing Medical University, National Clinical Research Center for Child Health \\ and Disorders, Ministry of Education Key Laboratory of Child Development and Disorders, Chongqing Key Laboratory of Pediatrics, Chongqing, \\ China; ${ }^{2}$ Department of Pediatric Intensive Care Unit Children's Hospital of Chongqing Medical University, National Clinical Research Center for \\ Child Health and Disorders, Ministry of Education Key Laboratory of Child Development and Disorders, Chongqing Key Laboratory of Pediatrics, \\ Chongqing, China; ${ }^{3}$ Department of Clinical Pharmacy, School of Pharmaceutical Sciences, Shandong University, Jinan, China \\ Contributions: (I) Conception and design: L Peng, W Zhao, Z Luo; (II) Administrative support: Z Luo; (III) Provision of study materials or patients: \\ L Peng, Y Chen, Z Luo; (IV) Collection and assembly of data: L Peng, Y Gao, G Zhang, X Tian; (V) Data analysis and interpretation: L Peng, H \\ Xu, Q Yu, J Cheng, Y Li, Q Li; (VI) Manuscript writing: All authors; (VII) Final approval of manuscript: All authors. \\ Correspondence to: Zhengxiu Luo. Department of Respiratory Medicine, Children's Hospital of Chongqing Medical University, Chongqing 401122, \\ China. Email: luozhengxiu816@163.com.
}

Background: Vancomycin trough concentrations are associated with clinical outcomes and drug adverse effects. This study investigates the effects of continuous venovenous hemofiltration $(\mathrm{CVVH})$ on vancomycin trough concentrations in critically ill children with a vancomycin dosage of 40-60 mg/ $\mathrm{kg} /$ day.

Methods: Children with steady-state vancomycin trough concentrations admitted to the pediatric intensive care unit (PICU) between January 2016 and December 2019 were retrospectively enrolled. Patients were divided into $\mathrm{CVVH}$ and non-CVVH groups according to treatment differences and renal function. Vancomycin trough concentrations were then compared between the groups, and risk factors for supratherapeutic trough concentrations $(>20 \mathrm{mg} / \mathrm{L})$ were analyzed with logistic regression.

Results: Of the 119 patients included, 35 were enrolled in the CVVH group and 84 in the non-CVVH group. Median vancomycin trough concentrations were significantly higher in the CVVH group than those in the non-CVVH group [14.9 (IQR =9.6-19.6) vs. 9.3 (IQR =7.0-13.4), $\mathrm{P}<0.001]$ and the proportion of therapeutic trough concentrations $(10-20 \mathrm{mg} / \mathrm{L})$ was similar between $\mathrm{CVVH}$ and non-CVVH groups (54.3\% vs. 39.3\%, $\mathrm{P}=0.133)$. However, $\mathrm{CVVH}$ therapy patients had a significantly higher proportion of supratherapeutic trough concentrations $(20.0 \%$ vs. $1.2 \%, \mathrm{P}=0.001)$ compared to the non-CVVH group. Multivariate analysis demonstrated that the Pediatric Risk of Mortality (PRISM) III score $\geq 28$ (OR =13.7; 95\% CI, 1.4-137.0; $\mathrm{P}=0.026$ ] was an independent risk factor for supratherapeutic trough concentrations in critically ill patients.

Conclusions: CVVH therapy affects vancomycin trough concentrations and is associated with supratherapeutic concentrations with a $40-60 \mathrm{mg} / \mathrm{kg} /$ day vancomycin dosage. PRISM III scores $\geq 28$ may serve as an independent risk factor for supratherapeutic trough concentrations in children receiving $\mathrm{CVVH}$ therapy.

Keywords: Vancomycin; continuous venovenous hemofiltration (CVVH); children; concentration

Submitted May 17, 2020. Accepted for publication Nov 22, 2020.

doi: $10.21037 /$ atm-20-4005

View this article at: http://dx.doi.org/10.21037/atm-20-4005 


\section{Introduction}

Vancomycin is a major glycopeptide antibiotic used in the treatment of most Gram-positive infections caused by methicillin-resistant Staphylococcus aureus (MRSA), methicillin-resistant Staphylococcus epidermidis (MRSE), and amoxicillin-resistant enterococci (1). It can be taken orally to treat pseudomembranous enterocolitis caused by Clostridium difficile (2). Vancomycin is currently recommended as an empirical antibiotic for pediatric patients with severe sepsis and is one of the most frequently used antibiotics in the intensive care unit (ICU) $(3,4)$. Studies have indicated that nearly $30 \%$ of patients had sepsis either on admission to or during their ICU stay (5), and $21 \%$ of children with severe sepsis had acute kidney injury (AKI) and progress to requiring renal replacement therapy $(6,7)$. Also, children with AKI and receiving continuous renal replacement therapy (CRRT) had high mortality $(42-50 \%)(8,9)$. Continuous venovenous hemofiltration $(\mathrm{CVVH})$ is a convective clearance CRRT modality that can remove middle molecular weight substances (500-5,000 Da) efficiently with a little impact on hemodynamics (10). As vancomycin has a molecular weight of $1445 \mathrm{Da}$, it can be excreted during CVVH therapy (1).

Vancomycin is bactericidal and exhibits time-dependent killing, which means that its antibacterial activity is pharmacokinetically dependent on the time that its serum concentration is above the minimum inhibitory concentration (MIC) (11). A pharmacodynamic study demonstrated that the area under the curve (AUC) ratio to the MIC $\geq 400$ was an optimal predictor of vancomycin efficacy (12). However, in clinical practice, determining the AUC with the trapezoidal rule requires multiple serum concentrations, and it may be difficult for pediatricians to obtain multiple blood samples from children. Therefore, vancomycin trough concentrations have been regarded as surrogate measures for AUC/MIC (13). Reaching therapeutic trough concentrations is one of the most important factors in critically ill patients' successful treatment outcomes (14). Both the Infectious Diseases Society of America (IDSA) and the Japanese Society of Chemotherapy recommend a vancomycin trough concentration of $10-20 \mathrm{mg} / \mathrm{L} ; 10-15 \mathrm{mg} / \mathrm{L}$ for uncomplicated infections and $15-20 \mathrm{mg} / \mathrm{L}$ for serious infections (including bacteremia, infective endocarditis, osteomyelitis, meningitis, and pneumonia) $(13,15)$. The Chinese Pharmacological Society recommends 10-15 $\mathrm{mg} / \mathrm{L}$ as the therapeutic trough concentration for adult patients and $10-20 \mathrm{mg} / \mathrm{L}$ for severe MRSA infections (16).
Lower vancomycin trough concentrations are associated with increased mortality and drug resistance, while higher concentrations can induce vancomycin-associated nephrotoxicity (17-20). Studies in both adults and children revealed that concentrations higher than $20 \mathrm{mg} / \mathrm{L}$ were associated with nephrotoxicity (17-19). Achieving the optimal vancomycin trough concentration without drug toxicity is undoubtedly important for critically ill patients receiving concurrent vancomycin and CVVH therapy. Some studies investigated CVVH therapy's effects on vancomycin trough concentrations in adult patients (21-23). A prospective observational study suggested that continuous infusion vancomycin (CIV) achieved target concentrations more rapidly and consistently when critically ill adult patients received CVVH treatment (23). Frazee et al. (21) demonstrated an inverse association between CVVH hemofiltration rates and serum vancomycin concentrations in adults; increases in hemofiltration rates significantly correlated with reductions in trough concentrations. While studies of adult patients provide important insight, few studies have explored the correlations between CVVH therapy and vancomycin trough concentrations in children. We conducted this retrospective study to investigate the effects of $\mathrm{CVVH}$ on vancomycin trough concentrations in critically ill children receiving vancomycin dosage of $40-60 \mathrm{mg} / \mathrm{kg} /$ day. We present the following article following the STROBE reporting checklist (available at: http://dx.doi.org/10.21037/atm-20-4005).

\section{Methods}

\section{Study site and study population}

This was a retrospective, observational cohort study conducted in the Children's Hospital of Chongqing Medical University, a 1,500-bed [40 pediatric intensive care unit (PICU) beds] tertiary teaching hospital in Chongqing, China, ranked among the top three domestic children's hospitals (rank list: http://top100.imicams.ac.cn/home). A total of 445 patients hospitalized in the PICU between January 2016 and December 2019 and receiving vancomycin therapy were retrospectively enrolled. The inclusion criteria were all of the following: (I) aged $<18$ years, (II) received vancomycin therapy with a dose of $40-60 \mathrm{mg} / \mathrm{kg} /$ day $>48$ hours, (III) either normal renal function or AKI (24), (IV) serum trough concentrations were obtained at steady-state conditions, $0.5 \pm 0.5$ hour before the fourth or fifth dose (13), (V) if patients had $>1$ episode of vancomycin treatment, only the first episode was considered. The exclusion criteria included 
any of the following: (I) patients with incomplete clinical information, (II) concomitant extracorporeal membrane oxygenation (ECMO) therapy (25), (III) the vancomycin trough concentration was measured before CVVH treatment, (IV) AKI without CVVH therapy. The study was conducted following the Declaration of Helsinki (as revised in 2013) and approved by the institutional review board of the Children's Hospital of Chongqing Medical University (Approval ID: 2020-191). Informed individual consent was waived owing to the retrospective design of the study.

\section{Data collection}

Trained staff extracted patient data from electronic medical records using a standard collection form. Along with demographic characteristics (age, sex, body weight), the source of infection, the severity of illness estimated by the Pediatric Risk of Mortality (PRISM) III scores (26), renal parameters (hemofiltration rates, blood flow rates, dialyzer used and urine output), vancomycin-related details (dose, frequency and duration, and concentrations), duration of hospital stay, duration of PICU stay, and clinical outcomes (survival/non-survival) were collected.

\section{Classifications and definitions}

Patients were divided into two subgroups: (I) a CVVH group in which patients received vancomycin and $\mathrm{CVVH}$ therapy concurrently due to AKI and (II) a Non-CVVH group in which patients with normal renal function received vancomycin therapy only. We defined AKI as urine output $<0.5 \mathrm{~mL} / \mathrm{kg} / \mathrm{h}$ for 6 hours (24), and the source of infection was defined based on clinical records and laboratory or radiographic results. The severity of illness at the time of extracting serum vancomycin trough concentration samples was assessed by the PRISM III score (26), with a higher score indicating increasing severity. A vancomycin trough concentration between $10-20 \mathrm{mg} / \mathrm{L}$ was defined as a therapeutic trough concentration following IDSA guideline recommendations. A level $<10 \mathrm{mg} / \mathrm{L}$ was defined as a subtherapeutic trough concentration and a level $>20 \mathrm{mg} / \mathrm{L}$ as a supratherapeutic trough concentration (13).

\section{Vancomycin therapy and trough concentrations}

All eligible children received vancomycin (Vianex S.A., Athens, Greece) intravenously at an empiric dose of $40-60 \mathrm{mg} / \mathrm{kg} /$ day divided every 6 hours (a maximum daily dose $<2,000 \mathrm{mg}$ ) as recommended by pediatric references and drug instructions $(27,28)$. The individualized initial daily dosage was determined by attending pediatric clinicians according to the severity of the disease and renal function. The dosage regimen was recommended by therapeutic drug monitoring (TDM), and the TDM target for the trough concentration was $10-20 \mathrm{mg} / \mathrm{L}$ (13). Vancomycin was infused in an appropriate normal saline $(0.9 \%)$ intravenous injection for over 60 minutes, and its use was based on suspected or documented source(s) of Gram-positive cocci infections. Blood samples of approximately $2 \mathrm{~mL}$ were obtained at steady-state conditions $0.5 \pm 0.5$ hours before the fourth or fifth dose to determine the trough concentration (13). Trough concentration analysis was performed using the chemiluminescence immunoassay method (Abbott Laboratories, Chicago, IL, USA), which has an analytical range of $0.0-100.0 \mathrm{mg} / \mathrm{L}$ with a between-run coefficient of variation of $<15 \%$ throughout the analytical range. A central laboratory was assigned to test all blood samples within 24 hours, and intra- and inter-batch quality control was performed following the China National Accreditation Service for Conformity Assessment standard.

\section{CVVH procedures}

All CVVH treatments were performed using a $0.7 \mathrm{~m}^{2}$ high flux polysulfone membrane filter AEF-07 (Asahi Kasei Medical, Tokyo, Japan). A double-lumen catheter was inserted into either the internal jugular, femoral, or subclavian veins depending on clinical circumstances, and post-dilution performed using the CVVH system. The blood flow rate was $3-5 \mathrm{~mL} / \mathrm{kg} / \mathrm{min}$, and the hemofiltration rate was $20-35 \mathrm{~mL} / \mathrm{kg} / \mathrm{h}$ for each patient. Sodium citrate was added to equipment to prevent blood coagulation.

\section{Outcomes}

The primary outcome was the effects of CVVH on vancomycin trough concentrations with a vancomycin dosage of $40-60 \mathrm{mg} / \mathrm{kg} /$ day, and the secondary outcome was risk factors for supratherapeutic trough concentrations in critically ill children.

\section{Statistical analysis}

Descriptive statistics were used to express the demographic data. The categorical variables were described as numbers (n) and percentages (\%), while the median with inter- 


\section{Page 4 of 9}

quartile ranges (IQRs) was used to summarize non-normally distributed variables. Distribution of non-normally variables was assessed using the Mann-Whitney $U$ test, and the chi-square or Fisher's exact test studied categorical data. Receiver operating characteristic analysis was employed to select the optimal cutoff point of PRISM III scores using the maximum Youden's index (29) and calculated sensitivity and specificity with $95 \%$ confidence intervals (CIs) for the optimal cutoff point. Univariate and multivariate logistic regression analyses were used to evaluate the correlation between possible factors and supratherapeutic trough concentrations, and odds ratios (ORs) and 95\% CIs were calculated. For the logistic regression analysis, PRISM III scores were converted into categorical variables according to the optimal cutoff value as defined by receiver operating characteristic curve analysis, and a $\mathrm{P}$ value $<0.05$ (two-sided) was considered significant. IBM SPSS Statistics 22 (SPSS Inc., Chicago, IL, USA) was used for all statistical analyses.

\section{Results}

\section{Enrollment}

During the study period, 445 patients were evaluated for eligibility, and 161 were excluded for lacking available steady-state trough concentrations. A further 79 were excluded for receiving other vancomycin dosing regimens, 32 as they had AKI but did not receive CVVH therapy, 30 for having incomplete clinical information, 18 for having vancomycin trough concentrations measured before $\mathrm{CVVH}$ therapy, and six for receiving concomitant ECMO therapy. The remaining 119 patients were included in the study, of whom 35 received vancomycin and CVVH therapy simultaneously (Figure 1).

\section{Basic characteristics and demographic data of $\mathrm{CVVH}$ and non-CVVH group patients}

Basic characteristics and demographic data of CVVH and non-CVVH group patients are presented in Table 1. The median vancomycin trough concentrations and PRISM III scores in the CVVH group were significantly higher than those in the non-CVVH group [14.9 (IQR =9.6-19.6) vs. 9.3 (IQR =7.0-13.4), $\mathrm{P}<0.001 ; 28.0$ (IQR =21.0-32.0) vs. 21.0 (IQR =15.0-24.0), $\mathrm{P}<0.001$, respectively]. Of all enrolled patients, 27 had PRISM III scores $\geq 28$ and 70.4\% (19/27) of these received CVVH therapies. Moreover, CVVH therapy patients had a significantly longer duration of PICU stay
Peng et al. Effects of CVVH on vancomycin trough concentrations

[17.0 (IQR =10.0-32.0) vs. 13.0 (IQR =7.3-20.5) days, $\mathrm{P}=0.012]$ and hospital stay [39.0 (IQR $=20.0-57.0)$ vs. 22.0 (IQR $=15.0-34.0$ ) days, $\mathrm{P}<0.001]$ when compared to nonCVVH groups. There were no remarkable differences in demographic data, initial vancomycin daily doses, duration of vancomycin therapy, infection sites, and mortality between the two groups $(\mathrm{P}>0.05)$.

\section{Vancomycin trough concentrations in $\mathrm{CVVH}$ and non- CVVH groups}

Figure 2 shows the distribution of vancomycin trough concentrations between CVVH and non-CVVH group patients. The proportion of therapeutic trough concentrations was similar between CVVH and nonCVVH groups $(54.3 \%$ vs. $39.3 \%, \mathrm{P}=0.133)$. However, $\mathrm{CVVH}$ therapy group patients had a significantly higher proportion of supratherapeutic trough concentrations than non-CVVH groups $(20.0 \%$ vs. $1.2 \%, \mathrm{P}=0.001)$ with current vancomycin dosing strategies. Also, $87.5 \%(7 / 8)$ of patients with supratherapeutic vancomycin trough concentrations were in the CVVH therapy group. As expected, our results showed a remarkably lower proportion of subtherapeutic trough concentrations in the CVVH therapy group when compared to non-CVVH groups $(25.7 \%$ vs. $59.5 \%$, $\mathrm{P}=0.001)$.

\section{Risk factors for supratherapeutic trough concentrations}

On bivariate analysis, CVVH therapy and PRISM III scores were related to supratherapeutic trough concentrations (Table 2). The receiver operating characteristic curve (0.87; 95\% CI: 0.77-0.96) supported PRISM III scores could serve as a tool to screen supratherapeutic trough concentrations and PRISM III scores $\geq 28$ could be an optimal cutoff point with $87.5 \%$ sensitivity and $82.0 \%$ specificity. Further multivariate analysis revealed that a PRISM III score $\geq$ of 28 was the only independent risk factor for supratherapeutic trough concentrations in critically ill children $(\mathrm{OR}=13.7$; 95\% CI: 1.4-137.0; P=0.026) (Table 3).

\section{Discussion}

To the best of our knowledge, this is the first study to investigate the effects of CVVH therapy on vancomycin trough concentrations with a vancomycin dosage of $40-60 \mathrm{mg} / \mathrm{kg} /$ day. While our results showed CVVH and non-CVVH patients had a similar proportion of 


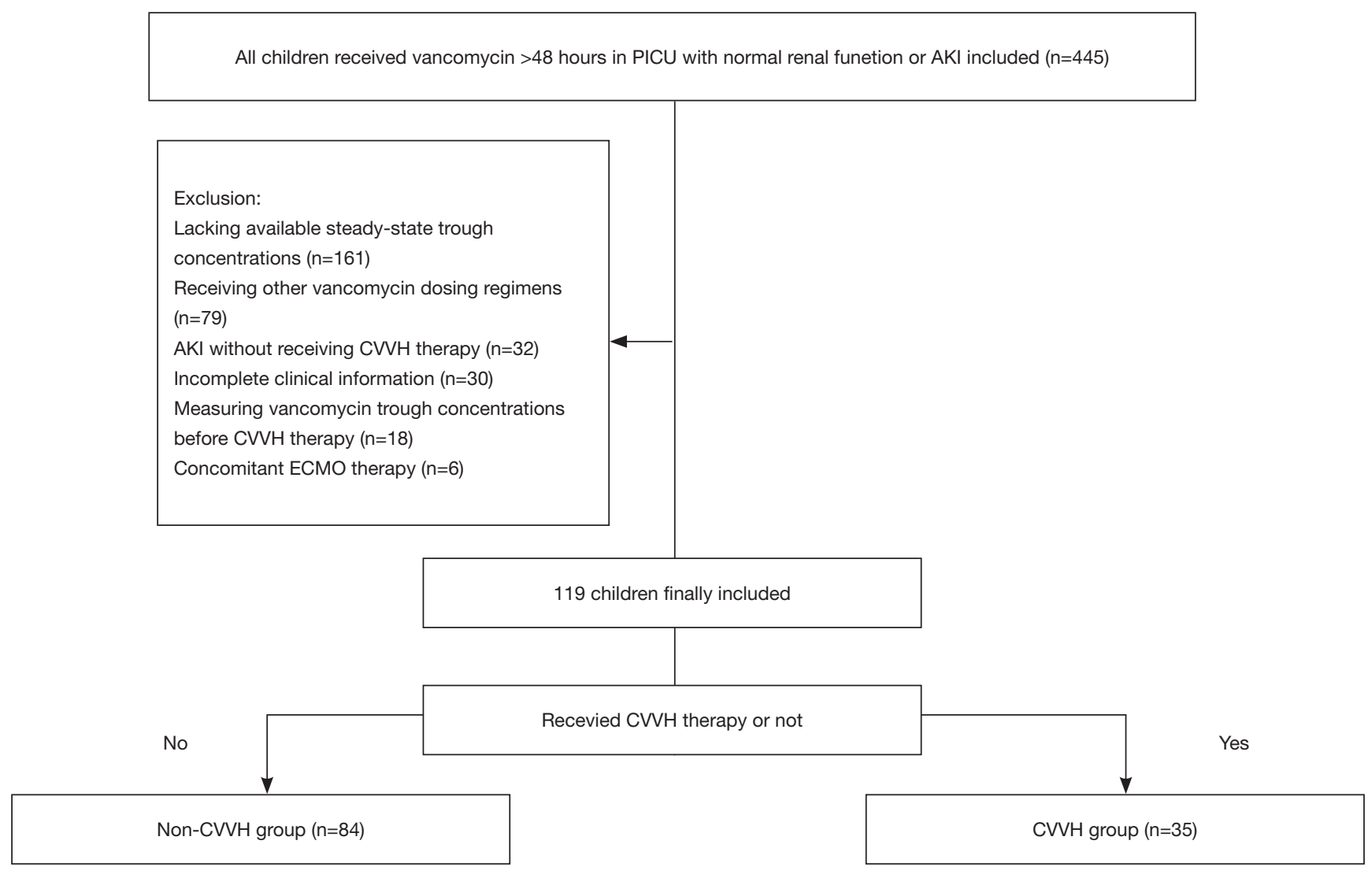

Figure 1 Flow chart of patient enrollment. PICU, pediatric intensive care unit; AKI, acute kidney injury; CVVH, continuous venovenous hemofiltration; ECMO, extracorporeal membrane oxygenation.

therapeutic trough concentrations, CVVH patients had a significantly higher proportion of supratherapeutic trough concentrations and a lower proportion of subtherapeutic trough concentrations when compared to the non-CVVH group. In contrast to our findings, Paciullo et al. (30) found CVVH therapy-induced subtherapeutic vancomycin concentrations in adult patients, and they recommended a higher vancomycin dosage for these patients. A possible explanation for this inconsistency could be in the significantly higher the average hemofiltration rate seen in the Paciullo study $(56 \mathrm{~mL} / \mathrm{kg} / \mathrm{h})$ in comparison to ours $(20-35 \mathrm{~mL} / \mathrm{kg} / \mathrm{h})$ as there is an inverse correlation between hemofiltration rates and vancomycin serum concentrations (21). In a prospective study, $\mathrm{Li}$ et al. (31) showed that CVVH clearance constituted $60-70 \%$ of total vancomycin clearance with a hemofiltration rate of $30-40 \mathrm{~mL} / \mathrm{kg} / \mathrm{h}$.

Nevertheless, more than $80 \%$ of vancomycin is eliminated in the urine within $24 \mathrm{~h}$ after administering a single dose in normal renal function patients (1). In this study, the hemofiltration rates of $20-35 \mathrm{~mL} / \mathrm{kg} / \mathrm{h}$ were lower than those in $\mathrm{Li}$ et al. (30-40 mL/kg/h) (31), leading to a lower percentage of vancomycin clearance. Therefore, vancomycin trough concentrations could be increased in CVVH therapy children due to vancomycin's reduced clearance.

Our results are consistent with recent studies, which showed that a dosage of $40-60 \mathrm{mg} / \mathrm{kg} / \mathrm{day}$ was inadequate to reach therapeutic vancomycin concentrations and suggested critically ill children receive higher dosages $(20,32)$. In our study's non-CVVH group, only $39.3 \%$ (33/84) of patients attained therapeutic vancomycin concentrations, and $60 \%(50 / 84)$ obtained subtherapeutic concentrations with the dosage of $40-60 \mathrm{mg} / \mathrm{kg} /$ day. However, our results also revealed CVVH therapy induced a higher proportion of supratherapeutic trough concentrations than non$\mathrm{CVVH}$ therapy, which indicated that children receiving $\mathrm{CVVH}$ therapy might require lower vancomycin dosages 
Table 1 Basic characteristics and demographic data of CVVH and non-CVVH therapy group patients

\begin{tabular}{|c|c|c|c|}
\hline Variables & CVVH group $(n=35)$ & Non-CVVH group $(n=84)$ & $P$ value \\
\hline Age (months), median (IQR) & $18.0(8.5-80.0)$ & $15.5(5.4-70.3)$ & 0.275 \\
\hline Male, n (\%) & $21(60.0)$ & $52(61.9)$ & 0.846 \\
\hline Body weight (kg), median (IQR) & $12.0(8.5-21.0)$ & $10.3(7.0-17.9)$ & 0.086 \\
\hline Initial daily dose (mg/kg/day), median (IQR) & $40.0(40.0-47.0)$ & $40.0(40.0-50.0)$ & 0.498 \\
\hline Duration of therapy (days), median (IQR) & $8.0(5.0-11.0)$ & $8.5(6.0-12.0)$ & 0.481 \\
\hline Trough concentrations (mg/L), median (IQR) & $14.9(9.6-19.6)$ & $9.3(7.0-13.4)$ & $<0.001$ \\
\hline PRISM III scores, median (IQR) & $28.0(21.0-32.0)$ & $21.0(15.0-24.0)$ & $<0.001$ \\
\hline \multicolumn{4}{|l|}{ Infection site ${ }^{a}$} \\
\hline Respiratory system, n (\%) & $25(71.4)$ & $66(78.6)$ & 0.403 \\
\hline Bacteremia, n (\%) & $16(45.7)$ & $32(38.1)$ & 0.440 \\
\hline Nervous system, n (\%) & $8(22.9)$ & $14(16.7)$ & 0.428 \\
\hline Skin and soft tissue, n (\%) & $6(17.1)$ & $6(7.1)$ & 0.099 \\
\hline Alimentary system, n (\%) & $4(11.4)$ & $2(2.4)$ & 0.061 \\
\hline Cardiovascular system, n (\%) & $1(2.9)$ & $3(3.6)$ & 1.000 \\
\hline Unknown, n (\%) & $0(0.0)$ & $2(2.4)$ & 1.000 \\
\hline
\end{tabular}

${ }^{a}$ The total percentage may $>100$ due to a patient having multiple infectious sites. CVVH, continuous venovenous hemofiltration; IQR, interquartile range; PRISM, pediatric risk of mortality; PICU, pediatric intensive care unit.

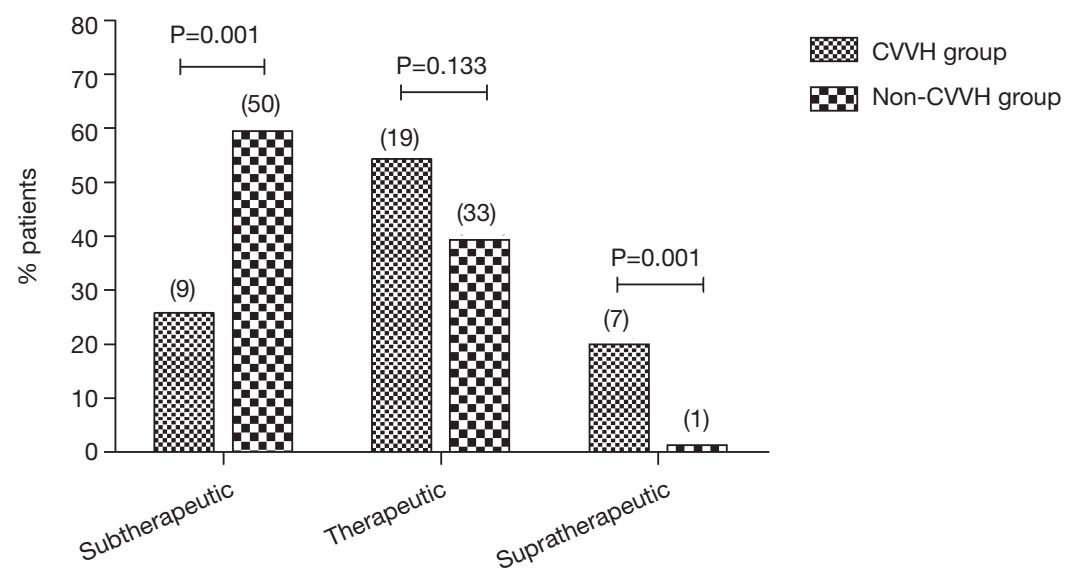

Figure 2 The distribution of vancomycin trough concentrations in CVVH and non-CVVH groups. The number in brackets above the columns reflects the total number of patients. CVVH, continuous venovenous hemofiltration. 
Table 2 Basic characteristics between different vancomycin trough concentrations

\begin{tabular}{|c|c|c|c|}
\hline Variables & Non-supratherapeutic $(n=111)$ & Supratherapeutic $(n=8)$ & $P$ value \\
\hline Male, n (\%) & $69(62.2)$ & $4(50.0)$ & 0.759 \\
\hline Body weight (kg), median (IQR) & $11.0(8.0-20.0)$ & $11.0(8.5-17.4)$ & 0.886 \\
\hline CVVH therapy, n (\%) & $28(25.2)$ & $7(87.5)$ & 0.001 \\
\hline Initial vancomycin daily dose (mg/kg/day), median (IQR) & $40.0(40.0-47.6)$ & $51.0(41.3-58.8)$ & 0.053 \\
\hline Duration of vancomycin therapy (days), median (IQR) & $8.0(6.0-12.0)$ & $7.5(4.3-9.8)$ & 0.171 \\
\hline
\end{tabular}

$\mathrm{CVVH}$, continuous venovenous hemofiltration; PRISM, pediatric risk of mortality; IQR, inter-quartile range.

Table 3 Logistic analysis of risk factors for supratherapeutic trough concentrations

\begin{tabular}{lcccc}
\hline \multirow{2}{*}{ Variables } & Univariate analysis & \multicolumn{2}{c}{ Multivariate analysis } \\
\cline { 2 - 3 } & OR $(95 \% \mathrm{Cl})$ & P value & OR $(95 \%$ Cl) & P value \\
\hline CVVH therapy & $20.8(2.4-176.1)$ & 0.005 & $0.6-67.3)$ & 0.111 \\
PRISM III scores $\geq 28$ & $31.9(3.7-273.6)$ & 0.002 & $13.7(1.4-137.0)$ & 0.026 \\
\hline
\end{tabular}

CVVH, continuous venovenous hemofiltration; PRISM, pediatric risk of mortality; $\mathrm{Cl}$, confidence interval; OR, odds ratio.

to obtain therapeutic concentrations than non-CVVH therapy patients. Undoubtedly, higher vancomycin daily dosages caused higher trough concentrations. Although no significant differences in the initial vancomycin daily dosage were found between supratherapeutic and nonsupratherapeutic groups $(\mathrm{P}>0.05)$, the results showed the median initial vancomycin dosage of the supratherapeutic group was $51 \mathrm{mg} / \mathrm{kg} /$ day, which was higher than that in the non-supratherapeutic group (40 mg/kg/day). Therefore, caution should be applied when adjusting vancomycin dosages in children receiving vancomycin and CVVH therapy concurrently. Moreover, close monitoring of vancomycin trough concentrations is highly warranted in children receiving vancomycin and CVVH therapy simultaneously.

We found that PRISM III scores $\geq 28$ increased the risk of reaching supratherapeutic trough concentrations nearly 13.7 -fold. Higher PRISM III scores indicate more serious conditions (26), and critically ill patients usually have organ dysfunctions, multi-drug interactions, and other therapeutic interventions, which may impact antimicrobial pharmacokinetics (33). In this study, 27 patients had PRISM III scores $\geq 28$, and 19 (70.4\%) had AKI and received CVVH therapy. We found that CVVH therapy was associated with supratherapeutic trough concentrations. This may indicate multiple organ dysfunctions, and CVVH therapy increases the accumulation and decreases the elimination of drugs, leading to higher vancomycin trough concentrations in critically ill patients. It has been widely acknowledged that higher vancomycin trough concentrations increased the risk of nephrotoxicity and that a concentration of higher than $20 \mathrm{mg} / \mathrm{L}$ was an independent risk factor for nephrotoxicity both in children and adult patients (17-19). The present study results support the observation that vancomycin trough concentrations should be monitored closely in children with PRISM III scores $\geq 28$. There are several limitations to this study. Firstly, its retrospective design means that it was difficult to control all the variables to avoid biases. Secondly, this was a single-center study, and the findings may be affected by the utilization of different institutional $\mathrm{CVVH}$ practices, including but not limited to hemofiltration rates, flow rates of the blood, dialyzer selection, and pre/post-dilution fluid. Thirdly, the lack of microbiological outcomes prevented us from analyzing the effect of subtherapeutic trough concentrations, and finally, we failed to assess supratherapeutic trough concentration effects on nephrotoxicity because of a small sample size. A larger multicenter prospective study is needed in the future 


\section{Page 8 of 9}

to investigate the association between vancomycin trough concentration and its associated nephrotoxicity in pediatric patients receiving $\mathrm{CVVH}$ therapy to validate our results.

\section{Conclusions}

CVVH therapy affects vancomycin trough concentrations and is associated with supratherapeutic trough concentrations with a 40-60 mg/kg/day vancomycin dosage. PRISM III scores $\geq 28$ may serve as an independent risk factor for supratherapeutic trough concentrations in CVVH therapy patients.

\section{Acknowledgments}

Funding: This work was supported by the National Key Clinical specialty (grant No. 2011-873).

\section{Footnote}

Reporting Checklist: The authors have completed the STROBE reporting checklist. Available at: http://dx.doi. org/10.21037/atm-20-4005

Data Sharing Statement: Available at http://dx.doi. org/10.21037/atm-20-4005

Peer Review File: Available at http://dx.doi.org/10.21037/ atm-20-4005

Conflicts of Interest: All authors have completed the ICMJE uniform disclosure form (available at: http://dx.doi. org/10.21037/atm-20-4005). The authors have no conflicts of interest to declare.

Ethical Statement: The authors are accountable for all aspects of the work in ensuring that questions related to the accuracy or integrity of any part of the work are appropriately investigated and resolved. The study was conducted in accordance with the Declaration of Helsinki (as revised in 2013). The study was approved by the institutional review board of the Children's Hospital of Chongqing Medical University (Approval ID: 2020-191) and individual consent for this retrospective analysis was waived.

Open Access Statement: This is an Open Access article distributed in accordance with the Creative Commons
Peng et al. Effects of CVVH on vancomycin trough concentrations

Attribution-NonCommercial-NoDerivs 4.0 International License (CC BY-NC-ND 4.0), which permits the noncommercial replication and distribution of the article with the strict proviso that no changes or edits are made and the original work is properly cited (including links to both the formal publication through the relevant DOI and the license). See: https://creativecommons.org/licenses/by-nc-nd/4.0/.

\section{References}

1. Rubinstein E, Keynan Y. Vancomycin revisited - 60 years later. Front Public Health 2014;2:217.

2. Levine DP. Vancomycin: a history. Clin Infect Dis 2006;42 Suppl 1:S5-S12.

3. Denny KJ, Cotta MO, Parker SL, et al. The use and risks of antibiotics in critically ill patients. Expert Opin Drug Saf 2016;15:667-78.

4. Rhodes A, Evans LE, Alhazzani W, et al. Surviving Sepsis Campaign: International Guidelines for Management of Sepsis and Septic Shock: 2016. Crit Care Med 2017;45:486-552.

5. Vincent JL, Marshall JC, Namendys-Silva SA, et al. Assessment of the worldwide burden of critical illness: the intensive care over nations (ICON) audit. Lancet Respir Med 2014;2:380-6.

6. Fitzgerald JC, Basu RK, Akcan-Arikan A, et al. Acute Kidney Injury in Pediatric Severe Sepsis: An Independent Risk Factor for Death and New Disability. Crit Care Med 2016;44:2241-50.

7. Zhang J, Tian J, Sun H, et al. How Does Continuous Renal Replacement Therapy Affect Septic Acute Kidney Injury? Blood Purif 2018;46:326-31.

8. Symons JM, Chua AN, Somers MJ, et al. Demographic characteristics of pediatric continuous renal replacement therapy: a report of the prospective pediatric continuous renal replacement therapy registry. Clin J Am Soc Nephrol 2007;2:732-8.

9. Al-Ayed T, Rahman NU, Alturki A, et al. Outcome of continuous renal replacement therapy in critically ill children: a retrospective cohort study. Ann Saudi Med 2018;38:260-8.

10. Maursetter L, Kight CE, Mennig J, et al. Review of the mechanism and nutrition recommendations for patients undergoing continuous renal replacement therapy. Nutr Clin Pract 2011;26:382-90.

11. Rybak MJ. The pharmacokinetic and pharmacodynamic properties of vancomycin. Clin Infect Dis 2006;42 Suppl 1:S35-9. 
12. Lodise TP, Graves J, Evans A, et al. Relationship between vancomycin MIC and failure among patients with methicillin-resistant Staphylococcus aureus bacteremia treated with vancomycin. Antimicrob Agents Chemother 2008;52:3315-20.

13. Liu C, Bayer A, Cosgrove SE, et al. Clinical practice guidelines by the infectious diseases society of america for the treatment of methicillin-resistant Staphylococcus aureus infections in adults and children. Clin Infect Dis 2011;52:e18-55.

14. Weiss SL, Fitzgerald JC, Balamuth F, et al. Delayed antimicrobial therapy increases mortality and organ dysfunction duration in pediatric sepsis. Crit Care Med 2014;42:2409-17.

15. Matsumoto K, Takesue Y, Ohmagari N, et al. Practice guidelines for therapeutic drug monitoring of vancomycin: a consensus review of the Japanese Society of Chemotherapy and the Japanese Society of Therapeutic Drug Monitoring. J Infect Chemother 2013;19:365-80.

16. Ye ZK, Chen YL, Chen K, et al. Therapeutic drug monitoring of vancomycin: a guideline of the Division of Therapeutic Drug Monitoring, Chinese Pharmacological Society. J Antimicrob Chemother 2016;71:3020-5.

17. Park SJ, Lim NR, Park HJ, et al. Evaluation of risk factors for vancomycin-induced nephrotoxicity. Int J Clin Pharm 2018;40:1328-34.

18. Dong MH, Wang JW, Wu Y, et al. Evaluation of body weight-based vancomycin therapy and the incidence of nephrotoxicity: a retrospective study in the northwest of China. Int J Infect Dis 2015;37:125-8.

19. Elyasi S, Khalili H, Dashti-Khavidaki S, et al. Vancomycininduced nephrotoxicity: mechanism, incidence, risk factors and special populations. A literature review. Eur J Clin Pharmacol 2012;68:1243-55.

20. Sridharan K, Al-Daylami A, Ajjawi R, et al. Vancomycin Use in a Paediatric Intensive Care Unit of a Tertiary Care Hospital. Paediatr Drugs 2019;21:303-12.

21. Frazee EN, Kuper PJ, Schramm GE, et al. Effect of continuous venovenous hemofiltration dose on achievement of adequate vancomycin trough concentrations. Antimicrob Agents Chemother 2012;56:6181-5.

22. Roberts DM, Roberts JA, Roberts MS, et al. Variability of antibiotic concentrations in critically ill patients receiving continuous renal replacement therapy: a multicentre pharmacokinetic study. Crit Care Med 2012;40:1523-8.
23. Sin JH, Newman K, Elshaboury RH, et al. Prospective evaluation of a continuous infusion vancomycin dosing nomogram in critically ill patients undergoing continuous venovenous haemofiltration. J Antimicrob Chemother 2018;73:199-203.

24. Khwaja A. KDIGO clinical practice guidelines for acute kidney injury. Nephron Clin Pract 2012;120:c179-84.

25. Yang CJ, Wu CW, Wu CC. Effect of Extracorporeal Membrane Oxygenation on the New Vancomycin Dosing Regimen in Critically Ill Patients Receiving Continuous Venovenous Hemofiltration. Ther Drug Monit 2018;40:310-4.

26. Pollack MM, Patel KM, Ruttimann UE. PRISM III: an updated Pediatric Risk of Mortality score. Crit Care Med 1996;24:743-52.

27. Robertson J, Shilkofski N. The Harriet Lane Handbook: A Manual for Pediatric House Officers. 17th ed. Philadelphia: Elsevier Mosby; 2005.

28. Taketomo CK, Hodding JH, Kraus DM. Pediatric Dosage Handbook. 14th ed. Hudson: Lexi-Comp; 2007.

29. Youden WJ. Index for rating diagnostic tests. Cancer 1950;3:32-5.

30. Paciullo CA, Harned KC, Davis GA, et al. Vancomycin clearance in high-volume venovenous hemofiltration. Ann Pharmacother 2013;47:e14.

31. Li Q, Liang F, Sang L, et al. Pharmacokinetics of and maintenance dose recommendations for vancomycin in severe pneumonia patients undergoing continuous venovenous hemofiltration with the combination of predilution and postdilution. Eur J Clin Pharmacol 2020;76:211-7.

32. Sosnin N, Curtis N, Cranswick N, et al. Vancomycin is commonly under-dosed in critically ill children and neonates. Br J Clin Pharmacol 2019;85:2591-8.

33. Scaglione F, Paraboni L. Pharmacokinetics/ pharmacodynamics of antibacterials in the Intensive Care Unit: setting appropriate dosing regimens. Int J Antimicrob Agents 2008;32:294-301.

Cite this article as: Peng L, Gao Y, Zhang G, Tian X, Xu H, Yu Q, Cheng J, Li Y, Li Q, Chen Y, Zhao W, Luo Z. Effects of continuous venovenous hemofiltration on vancomycin trough concentrations in critically ill children. Ann Transl Med 2021;9(3):224. doi: 10.21037/atm-20-4005 